\title{
ON THE GOLDBACH PROBLEM IN ALGEBRAIC NUMBER FIELDS AND THE POSITIVITY OF THE SINGULAR INTEGRAL
}

\author{
TAKUMI NoDA
}

\begin{abstract}
We show the positivity of the singular integral which arises in the Goldbach problem in algebaic number fields.
\end{abstract}

\section{Introduction}

In this paper our main purpose is to show the positivity of the singular integral under a sufficient condition. The singular integral is the generalized Dirichlet integral which appears in the coefficient of asymptotic formula in the Waring problem and the Goldbach problem in algebraic number fields (see Y. Wang [14]).

In $\$ 1$ and $\$ 2$ we shall define a singular integral which arises in the Goldbach problem in algebraic number fields and show the positivity as Theorem 1 . Here we notice that the positivity is not trivial if the algebraic number field $K$ has the complex conjugates. In $\S 3$ and $\S 4$ we shall derive an asymptotic formula as Theorem 2 following the generalized Vinogradov-Vaughan method introduced by Mitsui ([4], [5]). The asymptotic formula is a generalization of Sultanova [10] and Theorem 1 shows that the leading term of this formula has a positive coefficient.

\section{Statement of results}

Let $K$ be an algebraic number field of degree $n$. Let $K^{(q)}\left(q=1,2, \ldots, r_{1}\right)$ be the real conjugates of $K$ and $K^{(p)}, K^{\left(p+r_{2}\right)}\left(p=r_{1}+1, \ldots, r_{1}+r_{2}\right)$ be the complex conjugates of $K$ with $K^{\left(p+r_{2}\right)}=\bar{K}^{(p)}$. Let $\delta$ denote the different of $K$ and $D=N(b)$ (norm of $\delta$ ) the absolute value of the discriminant of $K$. Further, $h$ denotes the ideal class number of $K$ and $R$ the regulator of $K$. Let $\gamma$ be a number of $K$ and put $\mathfrak{b} \gamma=\mathfrak{b} / \mathfrak{a}$ with integral ideals $\mathfrak{a}$ and $\mathfrak{b}$ such that $(\mathfrak{a}, \mathfrak{b})=1$. We write this relation by $\gamma \rightarrow \mathfrak{a}$.

1991 Mathematics Subject Classification: 11P32, $11 R 47$

Received December 27, 1995; revised July 18, 1996. 
Let $\mu$ be a number of $K ; \mu$ also denotes an $n$-dimensional complex vector $\left(\mu^{(1)}, \mu^{(2)}, \ldots, \mu^{(n)}\right)$ with $\mu^{(i)} \in K^{(i)}(i=1,2, \ldots, n)$. More generally we consider any $n$-dimensional complex vector $\xi=\left(\xi_{1}, \xi_{2}, \ldots, \xi_{n}\right)$ with real $\xi_{q}(q=1,2$, $\left.\ldots, r_{1}\right)$ and complex $\xi_{p+r_{2}}=\xi_{p}\left(p=r_{1}+1, \ldots, r_{1}+r_{2}\right)$. We denote the set of $\xi$ by $E^{r_{1}, r_{2}}$. For $\xi \in E^{r_{1}, r_{2}}$, we write

$$
N(\xi)=\prod_{i=1}^{n} \xi_{i}, \quad S(\xi)=\sum_{j=1}^{n} \xi_{j} \quad \text { and } \quad E(\xi)=e^{2 \pi i S(\xi)} .
$$

Let $x(\xi)$ denote the $n$-dimensional real vector $x(\xi)=\left(X_{1}(\xi), X_{2}(\xi), \ldots, X_{n}(\xi)\right)$ with $X_{q}(\xi)=\xi_{q}, X_{p}(\xi)=\left(\xi_{p}+\xi_{p}\right) / 2$ and $X_{p+r_{2}}(\xi)=\left(\xi_{p}-\xi_{p}\right) / 2 \sqrt{-1}$. We denote the map from $E^{r_{1}, r_{2}}$ into $R^{n}$ such that the image of $\xi$ is $x(\xi)$ by $\phi$.

Let $D(t)(t>0)$ be a set of $\xi \in E^{r_{1}, r_{2}}$ such that $0<\xi_{q} \leq t\left(q=1, \ldots, r_{1}\right)$ and $\left|\xi_{p}\right| \leq t\left(p=r_{1}+1, \ldots, r_{1}+r_{2}\right)$. Regarding $X_{1}(\xi), \ldots, X_{n}(\xi)$ as variables we define an integral

$$
\Phi_{k}(z)=\frac{2^{r_{2}}}{\sqrt{D}} \int_{D(1)} E\left(z \xi^{k}\right) d x(\xi)
$$

where $k$ is a positive rational integer, $z \xi^{k}=\left(z_{1} \xi_{1}^{k}, \ldots, z_{n} \xi_{n}^{k}\right)$ with $z \in E^{r_{1}, r_{2}}$ and $d x(\xi)=d X_{1}(\xi) \cdots d X_{n}(\xi)$.

In the following we let $\mu$ be a totally positive integer and $a_{k}=\left(a_{k}^{(1)}, a_{k}^{(2)}, \ldots\right.$, $\left.a_{k}^{(n)}\right)(k=1,2, \ldots, s)$ be a point of $E^{r_{1}, r_{2}}$ which satisfy the condition:

$$
\begin{gathered}
a_{k}^{(i)} \in R, \quad a_{k}^{(i)}>0 \quad(i=1,2, \ldots, n ; k=1,2, \ldots, s), \\
0<a_{1}^{(i)} \leq a_{2}^{(i)} \leq \cdots \leq a_{s}^{(i)} \leq 1<1+c^{(i)}=a_{1}^{(i)}+a_{2}^{(i)}+\cdots+a_{s}^{(i)}
\end{gathered}
$$

with a positive constant $c^{(i)}$. We define a singular integral as follows:

$$
\Psi_{1}\left(\mu ; \lambda_{1}, \lambda_{2}, \ldots, \lambda_{\mathrm{s}}\right)=2^{r_{2}} \sqrt{D} \int_{R^{n}} \prod_{k=1}^{s} \Phi_{1}\left(\lambda_{k} z\right) E(-\mu z) d x(z)
$$

with

$$
\lambda_{k}=a_{k} \mu \quad(k=1,2, \ldots, s) .
$$

We shall prove the following theorem:

THEOREM 1. There is a positive constant $c_{1}$ which depends on $a_{k}^{(i)}(i=1$, $2, \ldots, n ; k=1,2, \ldots, s)$ such that

$$
\Psi_{1}\left(\mu ; \lambda_{1} \lambda_{2}, \ldots, \lambda_{s}\right) \geq \frac{c_{1}}{N(\mu)} .
$$

In this paper we call an integer $\omega$ of $K$ a prime number, if the principal ideal ( $\omega)$ is a prime ideal. Let $\Omega\left(\lambda_{k}\right)$ be a set of prime numbers $\omega_{k}$ of $K$ such that 
$0<\omega_{k}^{(q)} \leq \lambda_{k}^{(q)}\left(q=1,2, \ldots, r_{1}\right), \quad\left|\omega_{k}^{(p)}\right| \leq\left|\lambda_{k}^{(p)}\right| \quad\left(p=r_{1}+1, \ldots, r_{1}+r_{2}\right)$.

We define a sum $R\left(\mu ; \lambda_{1}, \lambda_{2}, \ldots, \lambda_{s}\right)$ as follows:

$$
R\left(\mu ; \lambda_{1}, \lambda_{2}, \ldots, \lambda_{s}\right)=\sum_{\mu=\omega_{1}+\cdots+\omega_{s}, \omega_{k} \in \Omega\left(\lambda_{k}\right)} \log N\left(\omega_{1}\right) \cdots \log N\left(\omega_{s}\right),
$$

where the sum is taken over all the $s$-tuples $\left(\omega_{1}, \omega_{2}, \ldots, \omega_{s}\right)$ of prime numbers such that

$$
\mu=\omega_{1}+\omega_{2}+\cdots+\omega_{s}, \quad \omega_{k} \in \Omega\left(\lambda_{k}\right) \quad(k=1,2, \ldots, s) .
$$

Then we have

THEOREM 2. Let $\mu$ be a totally positive integer of $K$ and $s$ be a rational integer with $s \geq 3$. Then

$$
\begin{aligned}
R\left(\mu ; \lambda_{1}, \lambda_{2}, \ldots, \lambda_{s}\right)= & \frac{\Psi_{1}\left(1 ; a_{1}, a_{2}, \ldots, a_{s}\right)}{W^{s}} \Im_{G}(\mu) \prod_{k=1}^{s} N\left(a_{k}\right) N(\mu)^{s-1} \\
& +O\left(\frac{N^{(s-1) n}}{(\log N)^{s+1}}\right)
\end{aligned}
$$

where $N=\max \left\{\left|\lambda_{s}^{(i)}\right|\right\}_{(1 \leq i \leq n)}, W=2^{r_{1}+r_{2}} \pi^{r_{2}} h R / \omega \sqrt{D}$ with $\omega$ the number of the roots of unity in $K$ and $\Im_{G}(\mu)$ is the singular series which is written as an infinite product:

$$
\widetilde{S}_{G}(\mu)=\prod_{\mathfrak{p} \mid \mu}\left(1+\frac{(-1)^{s}}{(N(\mathfrak{p})-1)^{s-1}}\right) \prod_{\mathfrak{p} \nmid \mu}\left(1+\frac{(-1)^{s+1}}{(N(\mathfrak{p})-1)^{s}}\right) .
$$

\section{Singular integral}

First, we recall a fundamental lemma for $\Phi_{k}(z)$.

Lemma 1 ([5] Theorem 4.6.1, [14] Lemma 5.3). For $k \geq 2$ we have

$$
\Phi_{k}(z) \ll \prod_{i=1}^{n} \min \left(1,\left|z_{j}\right|^{-\frac{1}{k}}\right) .
$$

In the case $k=1$, we have

$$
\Phi_{1}(z) \ll \prod_{i=1}^{r_{1}+r_{2}} \min \left(1,\left|z_{j}\right|^{-1}\right)
$$

If $s>2 k$

$$
\int_{\boldsymbol{R}^{n}}\left|\Phi_{k}(z)\right|^{s} d x(z)
$$

is convergent. 
We now prove the positivity of a singular integral with a linear restriction on the integral $\Phi_{k}(z)$.

Proof of Theorem 1. $\Psi_{1}\left(\mu ; \lambda_{1}, \lambda_{2}, \ldots, \lambda_{s}\right)$ is written as follows:

$$
\begin{aligned}
= & \frac{2^{r_{2}(1+s)}}{D^{\frac{s-1}{2}}} \prod_{q=1}^{r_{1}} \int_{-\infty}^{\infty} \prod_{k=1}^{s}\left\{\int_{0}^{1} \exp \left(2 \pi i \lambda_{k}^{(q)} z_{q} \xi_{q}\right) d X_{q}\left(\xi_{q}\right)\right\} \exp \left(-2 \pi i \mu^{(q)} z_{q}\right) d X_{q}(z) \\
& \times \prod_{p=r_{1}+1}^{r_{1}+r_{2}} \int_{-\infty}^{\infty} \int_{-\infty}^{\infty} \prod_{k=1}^{s}\left\{\iint_{\left|\xi_{p}\right| \leq 1} \exp \left(2 \pi i\left(\lambda_{k}^{(p)} z_{p} \xi_{p}+\bar{\lambda}_{k}^{(p)} \bar{z}_{p} \bar{\xi}_{p}\right)\right)\right. \\
& \left.d X_{p}(\xi) d X_{p+r_{2}}(\xi)\right\} \times \exp \left(-2 \pi i\left(\mu^{(p)} z_{p}+\bar{\mu}^{(p)} \bar{z}_{p}\right)\right) d X_{p}(z) d X_{p+r_{2}}(z) .
\end{aligned}
$$

We consider the factors of product $\prod_{q}$ and $\prod_{p}$ on the right hand side of (2.1). Firstly, the factor of product $\prod_{p}$ is

$$
\begin{aligned}
& \text { (2.2) } \int_{-\infty}^{\infty} \prod_{k=1}^{s}\left\{\int_{0}^{1} \exp \left(2 \pi i \lambda_{k}^{(q)} z_{q} \xi_{q}\right) d \xi_{q}\right\} \exp \left(-2 \pi i \mu^{(q)} z_{q}\right) d z_{q} \\
& =\frac{1}{\mu^{(q)}} \int_{-\infty}^{\infty} \prod_{k=1}^{s}\left\{\int_{0}^{1} \exp \left(2 \pi i a_{k}^{(q)} z_{q} \xi_{q}\right) d \xi_{q}\right\} \exp \left(-2 \pi i z_{q}\right) d z_{q} \\
& =\frac{1}{\mu^{(q)}} \int_{-\infty}^{\infty}\left\{\int_{0}^{1} \cdots \int_{0}^{1} e^{2 \pi i z_{q}\left(a_{1}^{(q)} \xi_{1}+a_{2}^{(q)} \xi_{2}+\cdots+a_{s}^{(q)} \xi_{s}\right)} d \xi_{1} d \xi_{2} \cdots d \xi_{s}\right\} e^{-2 \pi l z_{q}} d z_{q} \\
& =\frac{1}{\mu^{(q)} \prod_{k=1}^{s} a_{k}^{(q)}} \int_{-\infty}^{\infty}\left\{\int_{0}^{d_{1}^{(q)}} \int_{0}^{a_{2}^{(q)}} \cdots \int_{0}^{a_{s}^{(q)}} e^{2 \pi t z_{q}\left(u_{1}+u_{2}+\cdots+u_{s}\right)} d u_{1} d u_{2} \cdots d u_{s}\right\} \\
& e^{-2 \pi i z_{q}} d z_{q} .
\end{aligned}
$$

Then, putting $w=u_{1}+\cdots+u_{s}$, we have

$$
\begin{aligned}
= & \frac{1}{\mu^{(q)} \prod_{k=1}^{s} a_{k}^{(q)}} \int_{-\infty}^{\infty}\left\{\int_{A^{(q)}} e^{2 \pi i z_{q} w} d u_{1} d u_{2} \cdots d u_{s-1} d w\right\} e^{-2 \pi l z_{q}} d z_{q} \\
= & \frac{1}{\mu^{(q)} \prod_{k=1}^{s} a_{k}^{(q)}} \int_{-\infty}^{\infty}\left\{\int_{0}^{d_{1}^{(q)}+\cdots+d_{s}^{(q)}}\left(\int_{B^{(q)}} d u_{1} \cdots d u_{s-1}\right) e^{2 \pi i z_{q} w} d w\right\} \\
& e^{-2 \pi i z_{q}} d z_{q}
\end{aligned}
$$

where $A^{(q)}$ and $B^{(q)}$ are defined as follows: 


$$
\begin{aligned}
& A^{(q)}=\left\{\begin{array}{l|l}
\left(u_{1}, u_{2}, \ldots, u_{s-1}, w\right) \in R^{s} & \begin{array}{l}
0 \leq u_{1} \leq a_{1}^{(q)}, \ldots, 0 \leq u_{s-1} \leq a_{s-1}^{(q)} \\
0 \leq w \leq a_{1}^{(q)}+\ldots+a_{s}^{(q)} \\
0 \leq w-u_{1}-\ldots-u_{s-1} \leq a_{s}^{(q)}
\end{array}
\end{array}\right\}, \\
& B^{(q)}=\left\{\begin{array}{l|l}
\left(u_{1}, u_{2}, \ldots, u_{s-1}\right) \in R^{s-1} & \begin{array}{l}
0 \leq u_{1} \leq a_{1}^{(q)} \ldots, 0 \leq u_{s-1} \leq a_{s-1}^{(q)} \\
0 \leq w-u_{1}-\ldots-u_{s-1} \leq a_{s}^{(q)}
\end{array}
\end{array}\right\} .
\end{aligned}
$$

Now we put

$$
F_{0}^{(q)}(w)=\int_{B^{(q)}} d u_{1} d u_{2} \cdots d u_{s-1} .
$$

Then we have $F_{0}^{(q)}(w)=0$ for $w<0$ or $w>a_{1}^{(q)}+\cdots+a_{s}^{(q)}$ and $F_{0}^{(q)}(w)$ is a continuous function of $w$. Therefore, applying the theory of Fourier integrals, we find that $(2.2)$ is equal to

$$
\begin{aligned}
& \frac{1}{\mu^{(q)} \prod_{k=1}^{s} a_{k}^{(q)}} \int_{-\infty}^{\infty}\left\{\int_{-\infty}^{\infty} F_{0}^{(q)}(w) e^{2 \pi i z_{q} w} d w\right\} e^{-2 \pi k z_{q} \times 1} d z_{q} \\
= & \frac{1}{\mu^{(q)} \prod_{k=1}^{s} a_{k}^{(q)}} F_{0}^{(q)}(1) .
\end{aligned}
$$

In a similar way, the factor of product $\prod_{p}$ is

$$
\begin{aligned}
& (2.4) \int_{-\infty}^{\infty} \int_{-\infty}^{\infty} \prod_{k=1}^{s}\left\{\iint_{\left|\xi_{p}\right| \leq 1} \exp \left(2 \pi i\left(\lambda \lambda_{k}^{(p)} z_{p} \xi_{p}+\bar{\lambda}_{k}^{(p)} \bar{z}_{p} \bar{\xi}_{p}\right)\right) d X_{p}(\xi) d X_{p+r_{2}}(\xi)\right\} \\
& \times \exp \left(-2 \pi i\left(\mu^{(p)} z_{p}+\bar{\mu}^{(p)} \bar{z}_{p}\right)\right) d X_{p}(z) d X_{p+r_{2}}(z) \\
& =\frac{1}{2^{2} \mu^{(p)} \mu^{\left(p+r_{2}\right)} \prod_{k=1}^{s} a_{k}^{(p)} a_{k}^{\left(p+r_{2}\right)}} \int_{-\infty}^{\infty} \int_{-\infty}^{\infty}\left\{\int_{-\infty}^{\infty} \int_{-\infty}^{\infty} G^{(p)}(U, V) e^{2 \pi i(u U+v V)} d U d V\right\} \\
& \times e^{-2 \pi i(u \times 1+v \times 0)} d u d v \\
& =\frac{1}{2^{2} \mu^{(p)} \mu^{\left(p+r_{2}\right)} \prod_{k=1}^{s} a_{k}^{(p)} a_{k}^{\left(p+r_{2}\right)}} G^{(p)}(1,0),
\end{aligned}
$$

where

$$
G^{(p)}(U, V)=\int_{D^{(p)}} d x_{1} d x_{2} \cdots d x_{s-1} d y_{1} d y_{2} \cdots d y_{s-1}
$$

is a $2(s-1)$-fold integral with 
$D^{(p)}=\left\{\begin{array}{l|l}x_{1}, x_{2}, \ldots, x_{s-1} & \begin{array}{l}x_{1}^{2}+y_{1}^{2} \leq\left(a_{1}^{(p)}\right)^{2}, \ldots, x_{s-1}^{2}+y_{s-1}^{2} \leq\left(a_{s-1}^{(p)}\right)^{2} \\ y_{1}, y_{2}, \ldots, y_{s-1} \\ \left(U-x_{1}-\cdots-x_{s-1}\right)^{2}+\left(V+y_{1}+\cdots+y_{s-1}\right)^{2} \\ \leq\left(a_{s}^{(p)}\right)^{2}\end{array}\end{array}\right\}$

By (2.1), (2.3) and (2.4) we obtain

$$
\Psi_{1}\left(\mu ; \lambda_{1}, \lambda_{2}, \ldots, \lambda_{s}\right)=\frac{2^{r_{2}(s-1)} D^{\frac{1-s}{2}}}{N(\mu) \prod_{k=1}^{s} N\left(a_{k}\right)} \prod_{q=1}^{r_{1}} F_{0}^{(q)}(1) \prod_{p=r_{1}+1}^{r_{1}+r_{2}} G^{(p)}(1,0) .
$$

Here $F_{0}^{(q)}(1)$ and $G^{(q)}(1.0)$ denote the volumes of domains $B_{0}^{(q)}$ and $D_{0}^{(p)}$ in $(s-1)$ and 2(s-1)-dimensional euclidian space, where $B_{0}^{(q)}$ and $D_{0}^{(p)}$ are given as follows:

$$
\begin{aligned}
& B_{0}^{(q)}=\left\{\begin{array}{l|l}
\left(u_{1}, u_{2}, \ldots, u_{s-1}\right) \in R^{s-1} & \begin{array}{l}
0 \leq u_{1} \leq a_{1}^{(q)}, \ldots, 0 \leq u_{s-1} \leq a_{s-1}^{(q)} \\
0 \leq 1-u_{1}-\ldots-u_{s-1} \leq a_{s}^{(q)}
\end{array}
\end{array}\right\},
\end{aligned}
$$

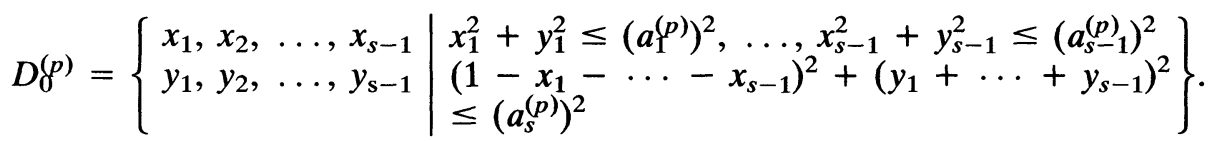

We shall show the existence of domains $B_{1}^{(q)}$ and $D_{1}^{(p)}$ such that $B_{1}^{(q)} \subset B_{0}^{(q)}$, $D_{1}^{(p)} \subset D_{0}^{(p)}$ and that the volumes of $B_{1}^{(q)}$ and $D_{1}^{(p)}$ are positive in each euclidian space. We now consider two cases to define $B_{1}^{(q)}$.

Case 1. $a_{1}^{(q)}+a_{2}^{(q)}+\cdots+a_{s-1}^{(q)}<1$. Let us define

$$
B_{1}^{(q)}=\left\{u_{1}, u_{2}, \ldots, u_{s-1} \mid a_{i}^{(q)}-\delta^{(q)} \leq u_{i} \leq a_{i}^{(q)} \quad(i=1,2, \ldots, s-1)\right\},
$$

where

$$
\delta^{(q)}=\min \left(a_{1}^{(q)}, c^{(q)} /(s-1)\right) .
$$

Case 2. $1 \leq a_{1}^{(q)}+a_{2}^{(q)}+\cdots+a_{s-1}^{(q)}$. Let us define $B_{1}^{(q)}=\left\{\begin{array}{l|l}u_{1}, u_{2}, \ldots, u_{s-1} & \begin{array}{l}a_{i}^{(q)}-h_{i}^{(q)} \leq u_{i} \leq a_{i}^{(q)}-h_{i}^{(q)}+\delta_{i}^{(q)} \\ (i=1,2, \ldots, s-1)\end{array}\end{array}\right\}$

with

$$
\begin{aligned}
& h_{i}^{(q)}=s_{i}^{(q)} c^{(q)} \\
& \delta_{i}^{(q)} \overline{h i}_{i}^{(q)}+s_{i}^{(q)}\left(1-a_{1}^{(q)}-\cdots-a_{s-1}^{(q)}\right),
\end{aligned}
$$

where we take positive constants $s_{i}^{(q)}(i=1,2, \ldots, s-1)$ which satisfy following conditions:

$$
\begin{aligned}
& s_{1}^{(q)}+s_{2}^{(q)}+\cdots+s_{s-1}^{(q)}=1 \\
& s_{i}^{(q)} c^{(q)} \leq a_{i}^{(q)}
\end{aligned}
$$


It is easy to see that such constants $s_{i}^{(q)}$ exist for all $q=1,2, \ldots, r_{1}$ and $B_{1}^{(q)} \subset B_{0}^{(q)}$ in each case. Then we have

$$
F_{0}^{(q)}(1) \geq \int_{B_{1}(q)} d u_{1} \cdots d u_{s-1}=\prod_{i=1}^{s-1} \delta_{i}^{(q)}
$$

Now we consider two cases to define $D_{1}^{(p)}$.

Case 1. Suppose $2 a_{s}^{(q)} \geq c^{(q)}$. Let us define

$D_{1}^{(p)}=\left\{\begin{array}{l|l}x_{1}, x_{2}, \ldots, x_{s-1} & a_{j}^{(p)}-s \delta^{(p)} /(s-1) \leq x_{j} \leq a_{J}^{(p)}-\delta^{(p)} \\ y_{1}, y_{2}, \ldots, y_{s-1} & 0 \leq y_{j} \leq \delta^{(p)} /(s-1)(j=1,2, \ldots, s-1)\end{array}\right\}$,

where

$$
\delta^{(p)}=\min \left(a_{1}^{(p)} / 2, c^{(p)} / 2 s\right)
$$

For $z=\left(x_{1}, x_{2}, \ldots, x_{s-1}, y_{1}, y_{2}, \ldots, y_{s-1}\right) \in D_{1}^{(p)}$, the coordinates $x_{1}, \ldots, x_{s-1}$, $y_{1}, \ldots, y_{s-1}$ satisfy the condition

$$
x_{j}^{2}+y_{j}^{2} \leq\left(a_{j}^{(p)}\right)^{2} \quad(j=1,2, \ldots, s-1) .
$$

In order to show $z \in D_{0}^{(p)}$, we shall prove

(2.8) $\left(1-x_{1}-x_{2}-\cdots-x_{s-1}\right)^{2}+\left(y_{1}+y_{2}+\cdots+y_{s-1}\right)^{2} \leq\left(a_{s}^{(p)}\right)^{2}$.

We consider two cases to show (2.8).

First, we take the point $z=\left(x_{1}, x_{2}, \ldots, d_{s-1}, y_{1}, y_{2}, \ldots, y_{s-1}\right) \in D_{1}^{(p)}$ with $x_{j}=a_{j}^{(p)}-\delta^{(p)}, y_{j}=\delta^{(p)} /(s-1)(j=1,2, \ldots, s-1)$.

Then we have

$$
\begin{aligned}
& \left(1-x_{1}-x_{2}-\cdots-x_{s-1}\right)^{2}+\left(y_{1}+y_{2}+\cdots+y_{s-1}\right)^{2} \\
= & \left\{a_{s}^{(p)}-c^{(p)}+(s-1) \delta^{(p)}\right\}^{2}+\left(\delta^{(p)}\right)^{2} \\
= & \left(a_{s}^{(p)}\right)^{2}-2 a_{s}^{(p)}\left\{c^{(p)}-(s-1) \delta^{(p)}\right\}+\left\{c^{(p)}-(s-1) \delta^{(p)}\right\}^{2}+\left(\delta^{(p)}\right)^{2} \\
\leq & \left(a_{s}^{(p)}\right)^{2}-c^{(p)}\left\{c^{(p)}-(s-1) \delta^{(p)}\right\}+\left\{c^{(p)}-(s-1) \delta^{(p)}\right\}^{2}+\left(\delta^{(p)}\right)^{2} \\
= & \left(a_{s}^{(p)}\right)^{2}-(s-1) \delta^{(p)} c^{(p)}+\left\{(s-1)^{2}+1\right\}\left(\delta^{(p)}\right)^{2} \\
< & \left(a_{s}^{(p)}\right)^{2}
\end{aligned}
$$

where the last inequality is followed by using $\delta^{(p)} \leq c^{(p)} / 2 s$.

Secondly, we take the point $z=\left(x_{1}, x_{2}, \ldots, x_{s-1}, y_{1}, y_{2}, \ldots, y_{s-1}\right) \in D_{1}^{(p)}$ with $x_{j}=a_{j}^{(p)}-s \delta^{(p)} /(s-1), y_{j}=\delta^{(p)} /(s-1)(j=1,2, \ldots, s-1)$. In a similar way we can see that the condition (2.8) is satisfied also on this point. Therefore the condition $(2.8)$ is satisfied on all points of $D_{1}^{(p)}$.

Case 2. Suppose $c^{(p)}>2 a_{s}^{(p)}$. Let $t_{j}^{(p)}$ be positive constants which satisfy the following conditions: 


$$
\begin{aligned}
& t_{1}^{(p)}+t_{2}^{(p)}+\cdots+t_{s-1}^{(p)}=1, \quad 0<t_{1}^{(p)}<t_{2}^{(p)}<\cdots<t_{s-1}^{(p)}<1, \\
& t_{j}^{(p)} \leq s a_{j}^{(p)} /(s+1) \quad(j=1,2, \ldots, s-1) .
\end{aligned}
$$

We define

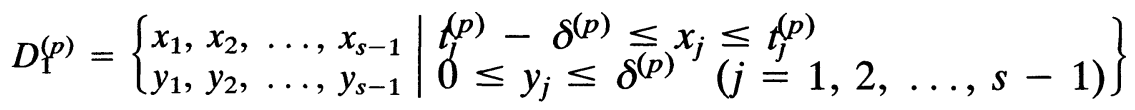

with

$$
\delta^{(p)}=\min \left(a_{1}^{(p)} /(s+1), t_{1}^{(p)}\right) .
$$

Under the condition $c^{(p)}>2 a_{s}^{(p)}$ such constants $t_{j}^{(p)}$ exist for all $p=r_{1}+1, \ldots$, $r_{1}+r_{2}$ and we see that $D_{1}^{(p)} \subset D^{(p)}$. Then we have

$$
D^{(p)}(1,0) \geq \int_{D_{1}^{(p)}} d x_{1} \cdots d x_{s-1} d y_{1} \cdots d y_{s-1} \geq\left\{\delta^{(p)} /(s-1)\right\}^{2(s-1)} .
$$

By (2.5), (2.6) and (2.9) we have

$$
\begin{gathered}
\Psi_{1}\left(\mu ; \lambda_{1}, \lambda_{2}, \ldots, \lambda_{s}\right) \geq \frac{2^{r_{2}(s-1)} D^{\frac{1-s}{2}}}{N(\mu) \prod_{k=1}^{s} N\left(a_{k}\right)} \prod_{q=1}^{r_{1}}\left\{\prod_{\iota=1}^{s-1} \delta_{i}^{(q)}\right\} \\
\prod_{p=r_{1}+1}^{r_{1}+r_{2}}\left\{\delta^{(p)} /(s-1)\right\}^{2(s-1)} .
\end{gathered}
$$

Now we consider the constant $c_{1}$ defined by

$$
c_{1}=\frac{2^{r_{2}(s-1)} D^{\frac{1-s}{2}}}{\prod_{k=1}^{s} N\left(a_{k}\right)} \prod_{q=1}^{r_{1}}\left\{\prod_{l=1}^{s-1} \delta_{i}^{(q)}\right\} \prod_{p=r_{1}+1}^{r_{1}+r_{2}}\left\{\delta^{(p)} /(s-1)\right\}^{2(s-1)},
$$

which allow us to establish Theorem 1.

\section{Farey dissection}

Let $\left\{\delta_{1}, \delta_{2}, \ldots, \delta_{n}\right\}$ be a basis of $\mathrm{b}^{-1}$. We put

$$
z_{J}=x_{1} \delta_{1}^{(j)}+x_{2} \delta_{2}^{(j)}+\ldots+x_{n} \delta_{n}^{(j)} \quad(j=1,2, \ldots, n)
$$

for real numbers $x_{1}, x_{2}, \ldots, x_{n}$ and define a set $E$ of $z=\left(z_{1}, z_{2}, \ldots, z_{n}\right)$ as follows:

$$
E=\left\{z \mid z_{J}=\sum_{i=1}^{n} x_{i} \delta_{i}^{(j)},-1 / 2<x_{i} \leq 1 / 2 \quad(j=1,2, \ldots, n)\right\}
$$

Let $H$ and $T$ be real numbers such that

$$
H>2 D T \quad \text { and } \quad T>1,
$$


and let $\Gamma$ be a set of numbers $\gamma$ of $K$ such that $\gamma \in E$ and $\gamma \rightarrow \mathfrak{a}$ with $N(\mathfrak{a}) \leq T^{n}$. In the following we let $\gamma_{1}$ be a number of $K$ and $N(\max (|z|, T))=\prod_{i=1}^{n} \max \left(\left|z_{i}\right|\right.$, $T)$.

Now we define divisions of $E$ in two ways.

(i) For every $\gamma \in \Gamma$ with $\gamma \rightarrow \mathfrak{a}$ we define a subset $E_{\gamma}$ of $E$ by $E_{\gamma}=\left\{z \in E \mid \exists \gamma_{1} \equiv \gamma\left(\bmod b^{-1}\right)\right.$ such that $\left.N\left(\max \left(H\left|z-\gamma_{1}\right|, T^{-1}\right)\right) \leq N(\mathfrak{a})^{-1}\right\}$ and put

$$
E^{0}=E-\cup_{\gamma \in \Gamma} E_{\gamma}
$$

(ii) For every $\gamma \in \Gamma$ with $\gamma \rightarrow \mathfrak{a}$ we define a subset $B_{\gamma}$ of $E$ by

$$
B_{\gamma}=\left\{z \in E \mid \begin{array}{l}
\exists \gamma_{1} \equiv \gamma\left(\bmod \delta^{-1}\right) \text { such that } \\
\left|z_{j}-\gamma_{1}^{(j)}\right| \leq T^{n-1} / H \quad(j=1,2, \ldots, n)
\end{array}\right\}
$$

and put

$$
B^{0}=E-\cup_{\gamma \in \Gamma} B_{\gamma} .
$$

These division of $E$ depend on the pair $(H, T)$. We shall call these divisions Farey dissection of $E$ with respect to $(H, T)$. The Farey dissection (i) was introduced by Siegel [8] and the Farey dissection (ii) by Mitsui ([4] §4).

Now we take positive constants $\sigma, \sigma_{1}, \sigma_{2}, u$ and $v$ such that

$$
\begin{aligned}
& \sigma \geq 5, \sigma_{2}>v, u-1 \geq \sigma, \\
& \min \left(\sigma_{1}-v, \sigma_{1}-v+v / n-1\right) \geq \sigma+u+v / n, \\
& \min \left(\sigma_{2}, v / n, \sigma_{1}-1,(u-v) / n-1\right) \geq \sigma+2, \\
& \min \left(\sigma_{2}, v / n, \sigma_{1}-1\right)-2 r-20 \geq 4 \sigma \quad\left(r=r_{1}+r_{2}-1\right) .
\end{aligned}
$$

We put

$$
N=\max \left\{\left|\lambda_{s}^{(i)}\right|\right\}_{(1 \leq i \leq n)}
$$

and

$$
H=N /(\log N)^{\sigma_{1}}, \quad T=(\log N)^{\sigma_{2}} .
$$

The following Lemma 2 was proved by Siegel and Lemma 3 is due to Mitsui.

LEMmA 2(Siegel [9]). If $\gamma_{1}$ and $\gamma_{2}$ belong to $\Gamma$ and $\gamma_{1} \neq \gamma_{2}$, then we have

$$
E_{\gamma_{1}} \cap E_{\gamma_{2}}=\varnothing \text {. }
$$

Lemma 3(Mistui [4] \$4). We have

$$
B_{\gamma} \supset E_{\gamma}, \quad B^{0} \subset E^{0}
$$

and if $\gamma_{1}$ and $\gamma_{2}$ belong to $\Gamma$ and $\gamma_{1} \neq \gamma_{2}$, then we have 


$$
B_{\gamma_{1}} \cap B_{\gamma_{2}}=\emptyset \text {. }
$$

\section{Asymptotic formula}

Let $\mu$ be a totally positive integer of $K$ with sufficiently large norm $N(\mu)$. For $z \in E$ we put

$$
S\left(z ; \lambda_{k}\right)=\sum_{\omega_{k} \in \Omega\left(\lambda_{k}\right)} E\left(\omega_{k} z\right) \log N\left(\omega_{k}\right) \quad(k=1,2, \ldots, s) .
$$

Then the integral

$$
\begin{aligned}
& \int_{-\frac{1}{2}}^{\frac{1}{2}} \cdots \int_{-\frac{1}{2}}^{\frac{1}{2}} \prod_{k=1}^{s} S\left(z ; \lambda_{k}\right) E(-\mu z) d x_{1} d x_{2} \cdots d x_{n} \\
& =2^{r_{2}} \sqrt{D} \int_{\phi(E)}^{s} \prod_{k=1}^{s} S\left(z ; \lambda_{k}\right) E(-\mu z) d x(z)
\end{aligned}
$$

is equal to $R\left(\mu ; \lambda_{1}, \lambda_{2}, \ldots, \lambda_{s}\right)$. To get an asymptotic formula of $R\left(\mu ; \lambda_{1}, \lambda_{2}, \ldots\right.$ $\left.\lambda_{s}\right)$ we shall quote the estimate of $S\left(z ; \lambda_{k}\right)$ in Mitsui [5]. In the case of rational field, we can find the estimate in Vaughan [11].

First, for any totally positive unit $\eta$ we have

$$
R\left(\eta \mu ; \eta \lambda_{1}, \eta \lambda_{2}, \ldots, \eta \lambda_{s}\right)=R\left(\mu ; \lambda_{1}, \lambda_{2}, \ldots, \lambda_{s}\right) .
$$

The theory of units allow us to take a totally positive unit $\eta_{0}$ satisfying

$$
c^{\prime} N(v)^{\frac{1}{n}}<\left|\eta_{0}^{(i)} \mu^{(i)}\right|<c^{\prime \prime} N(v)^{\frac{1}{n}} \quad(i=1,2, \ldots, n) .
$$

Taking $\eta_{0} \mu$ instead of $\mu$, we shall assume that $\mu$ in (4.1) satisfies the inequalities

$$
c^{\prime} N(v)^{\frac{1}{n}}<\left|\mu^{(i)}\right|<c^{\prime \prime} N(v)^{\frac{1}{n}} \quad(i=1,2, \ldots, n) .
$$

Then $N=\max \left\{\left|\lambda_{s}^{(i)}\right|\right\}_{(1 \leq i \leq n)}$ is sufficiently large and the inequalities

$$
c N<\left|\lambda_{k}^{(i)}\right| \leq N \quad(i=1,2, \ldots, n ; k=1,2, \ldots, s)
$$

are satisfied.

Lemma 4. Let $z$ be a point of E. For a positive constant $b$ we put

$$
\phi\left(B_{0}\right)=\left\{x(z) \in R^{n}|\quad| z_{i} \mid \leq(\log N)^{b} / N \quad(i=1,2, \ldots, n)\right\}
$$

Then we have

$$
\begin{aligned}
& 2^{r_{2}} \sqrt{D} \int_{\phi\left(B_{0}\right)} \prod_{k=1}^{s} \Psi_{1}\left(\lambda_{k} z\right) E(-\mu z) d x(z) \\
& =\Psi_{1}\left(\mu ; \lambda_{1}, \lambda_{2}, \ldots, \lambda_{s}\right)+O\left(1 / N^{n}(\log N)^{b(s-2)}\right) .
\end{aligned}
$$

Proof. By Lemma 1 we have 


$$
\begin{aligned}
\Psi_{1}\left(\lambda_{k} z\right) & \ll \prod_{j=1}^{r_{1}+r_{2}} \min \left(1,\left|\lambda_{k}^{(j)} z j\right|^{-1}\right) \\
& \ll N^{-\left(r_{1}+r_{2}\right)} \prod_{j=1}^{r_{1}+r_{2}} \min \left(N,\left|z_{j}\right|^{-1}\right) .
\end{aligned}
$$

Hence

$$
\begin{aligned}
& \Psi_{1}\left(\mu ; \lambda_{1}, \lambda_{2}, \ldots, \lambda_{s}\right)-2^{r_{2}} \sqrt{D} \int_{\phi\left(B_{0}\right)} \prod_{k=1}^{s} \Psi_{1}\left(\lambda_{k} z\right) E(-\mu z) d x(z) \\
= & 2^{r_{2}} \sqrt{D} \int_{R^{n_{-}-\phi\left(B_{0}\right)}} \prod_{k=1}^{s} \Psi_{1}\left(\lambda_{k} z\right) E(-\mu z) d x(z) \\
\ll & N^{-\left(r_{1}+r_{2}\right) s} \int_{R^{n}-\phi\left(B_{0}\right)}\left\{\prod_{j=1}^{r_{1}+r_{2}} \min \left(N,\left|z_{j}\right|^{-1}\right)\right\}^{s} d x(z) .
\end{aligned}
$$

Here, we see

$$
\begin{aligned}
& \int_{(\log N)^{b} / N}^{\infty} \min \left(N,|x|^{-1}\right)^{s} d x \ll \frac{N^{(s-1)}}{(\log N)^{b(s-1)}}, \\
& \int_{(\log N)^{b / N}}^{\infty} \int_{0}^{2 \pi} \min \left(N,|r|^{-1}\right)^{s} r d \theta d r \ll \frac{N^{(s-2)}}{(\log N)^{b(s-2)}}
\end{aligned}
$$

thus we obtain (4.3).

Under the notations of $\$ 3$, we quote the following Theorems:

Theorem A([4] Theorem 5.1, [5] Theorem 6.6.2).' If $z$ belongs to $E^{0}$,

$$
S(z, \lambda) \ll \frac{N^{n}}{(\log N)^{\sigma}} .
$$

THEOREM B([5] Theorem 6.2.1). : Let $\gamma_{0}$ be a number of $K$ such that

$$
\begin{aligned}
& \gamma_{0} \equiv \gamma \quad\left(\bmod \delta^{-1}\right), \\
& \left|z-\gamma_{0}^{(i)}\right| \leq T^{n-1} / H \quad(i=1,2, \ldots, n) .
\end{aligned}
$$

Then for $z \in B_{\gamma} \quad(\gamma \rightarrow \mathfrak{a})$,

$$
S(z, \lambda)=\frac{w \sqrt{\mathrm{D}} \mu(\mathfrak{a}) N(\lambda)}{2^{r_{1}+r_{2}} \pi^{r_{2}} h \varphi(\mathfrak{a}) R} \Phi_{1}\left(\lambda\left(z-\gamma_{0}\right)\right)+O\left(\frac{N^{n}}{(\log N)^{a-b+1}}\right)
$$

where

$$
b=(n-1) \sigma_{2}+\sigma_{1}, \quad a>b,
$$

and

$$
\mu(\mathfrak{a})=\sum_{\gamma \rightarrow \mathfrak{a}, \gamma_{i \bmod \mathfrak{o}^{-1}}} E(\gamma), \quad \varphi(\mathfrak{a})=\sum_{\gamma \rightarrow \mathfrak{a}, \gamma \bmod \mathfrak{b}^{-1}} E(\rho \gamma) \text { with } \rho \in \mathfrak{a}
$$


Now we prove the Theorem 2 .

Proof of Theorem 2. By the Farey dissection of $E$ defined in \$3, we have

$$
\begin{aligned}
R\left(\mu ; \lambda_{1}, \lambda_{2}, \ldots, \lambda_{s}\right)= & 2^{r_{2}} \sqrt{D}\left\{\int_{\phi\left(B^{0}\right)}+\sum_{\gamma \in \Gamma} \int_{\phi\left(B_{\gamma}\right)}\right\} \\
& \prod_{k=1}^{s} S\left(z ; \lambda_{k}\right) E(-\mu z) d x(z) .
\end{aligned}
$$

We shall estimate the right hand side of (4.4) on $\phi\left(B^{0}\right)$ and $\phi\left(B_{\gamma}\right)(\gamma \in \Gamma)$ respectively;

Let us consider the integral on $\phi\left(B^{0}\right)$. By Theorem A we have

$$
\begin{aligned}
& R\left(\mu ; \lambda_{1}, \lambda_{2}, \ldots, \lambda_{s}\right)=2^{r_{2}} \sqrt{D} \int_{\phi\left(B^{0}\right)} \prod_{k=1}^{s} S\left(z ; \lambda_{k}\right) E(-\mu z) d x(z) . \\
\ll & \frac{N^{(s-2)}}{(\log N)^{b(s-2)}} \int_{\phi(E)}\left|S\left(z ; \lambda_{s}\right)\right|^{2} d x(z) .
\end{aligned}
$$

Applying Parseval's identity and the prime ideal theorem, we find that this quantity is equal to

$$
\begin{aligned}
& \frac{N^{(s-2)}}{(\log N)^{b(s-2)}} \sum_{\omega \in \Omega\left(\lambda_{k}\right)} \log ^{2} N(\omega) \\
\ll & \frac{N^{(s-2)}}{(\log N)^{b(s-2)}} N^{n} \log N .
\end{aligned}
$$

Thus we have

$$
2^{r_{2}} \sqrt{D} \int_{\phi\left(B^{0}\right)} \prod_{k=1}^{s} S\left(z ; \lambda_{k}\right) E(-\mu z) d x(z) \ll \frac{N^{(s-1)}}{\left(\log N^{(s+1)}\right)}
$$

Now we look at the integral on $\phi\left(B_{\gamma}\right)(\gamma \in \Gamma)$. By Theorem B we have

$$
\begin{aligned}
& 2^{r_{2}} \sqrt{D} \int_{\phi\left(B_{\gamma}\right)} \prod_{k=1}^{s} S\left(z ; \lambda_{k}\right) E(-\mu z) d x(z) \\
& =\frac{2^{r_{2}} \sqrt{D} \mu(\mathfrak{a})^{s}}{W^{s} \varphi(\mathfrak{a})^{s}} \prod_{k=1}^{s} N\left(\lambda_{k}\right) \int_{\phi\left(B_{\gamma}\right)} \prod_{k=1}^{s} \Phi_{1}\left(\lambda_{k}\left(z-\gamma_{0}\right)\right) E(-\mu z) d x(z) \\
& \quad+O\left(\frac{N^{s n}}{(\log N)^{a-b+1}}\right) \int_{\phi\left(B_{\gamma}\right)}|E(-\mu z)| d x(z) .
\end{aligned}
$$

By putting $z$ instead of $z-\gamma_{0}$ and applying the estimate

$$
\int_{\phi\left(B_{\gamma}\right)} d x(z) \ll \frac{(\log N)^{b n}}{N^{n}}
$$


on the error term, we see that this is equal to

$$
\begin{array}{r}
\frac{2^{r_{2}} \sqrt{D} \mu(\mathfrak{a})^{s}}{W^{s} \varphi(\mathfrak{a})^{s}} \prod_{k=1}^{s} N\left(\lambda_{k}\right) E(-\mu \gamma) \int_{\phi\left(B_{\gamma}\right)} \prod_{k=1}^{s} \Phi_{1}\left(\lambda_{k}(z)\right) E(-\mu z) d x(z) \\
+O\left(N^{(s-1) n} /(\log N)^{a-b(n+1)+1}\right)
\end{array}
$$

with

$$
\phi\left(B_{\gamma}\right)=\left\{x(z) \in R^{n}|\quad| z_{j} \mid \leq(\log N)^{b} / N \quad(j=1,2, \ldots, n)\right\} .
$$

Taking summation of the both sides of (4.6) over all $\gamma \in \Gamma$, and together with the estimate

$$
\sum_{\gamma \in \Gamma} 1 \ll \sum_{N(\mathfrak{a}) \leq T^{n}} N(\mathfrak{a}) \leq T^{2 n}
$$

we have

$$
\begin{gathered}
\sum_{\gamma \in \Gamma} 2^{r_{2}} \sqrt{D} \int_{\phi\left(B_{\gamma}\right)} \prod_{k=1}^{s} S\left(z ; \lambda_{k}\right) E(-\mu z) d x(z) \\
=\frac{2^{r_{2}} \sqrt{D}}{W^{s}} \prod_{k=1}^{s} N\left(\lambda_{k}\right) \sum_{\gamma \in \Gamma} \frac{\mu(\mathfrak{a})^{s}}{\varphi(\mathfrak{a})^{s}} E(-\mu \gamma) \int_{\phi\left(B_{\gamma}\right)} \prod_{k=1}^{s} \Phi_{1}\left(\lambda_{k} z\right) E(-\mu z) d x(z) \\
+O\left(N^{(s-1) n} /(\log N)^{a-b(n+1)+1-2 n \sigma_{2}}\right) .
\end{gathered}
$$

By Lemma 4, we find the right hand side of (4.7) is

(4.8) $\frac{2^{r_{2}} \sqrt{D}}{W^{s}} \prod_{k=1}^{s} N\left(\lambda_{k}\right) \sum_{\gamma \in \Gamma} \frac{\mu(\mathfrak{a})^{s}}{\varphi(\mathfrak{a})^{s}} E(-\mu \gamma) \Psi_{1}\left(\mu ; \lambda_{1}, \lambda_{2}, \ldots, \lambda_{s}\right)+O\left(\frac{N^{(s-1) n}}{(\log N)^{s+1}}\right)$

with

$$
a \geq b(n+1)+1-2 n \sigma_{2}+s+1
$$

Now we apply the following property of the singular series $\widetilde{\Im}_{G}(\mu)$ (see Rademacher [7], Mitsui ([4] § 10)):

$$
\begin{aligned}
\mathfrak{S}_{G}(\mu) & =\sum_{\mathfrak{a}} \frac{\mu(\mathfrak{a})^{s}}{\varphi(\mathfrak{a})^{s}} \sum_{\gamma \in \Gamma, \gamma \bmod \mathfrak{b}^{-1}} E(-\mu \gamma) \\
& =\sum_{N(\mathfrak{a}) \leq T^{n}} \frac{\mu(\mathfrak{a})^{s}}{\varphi(\mathfrak{a})^{a}} \sum_{\gamma \in \Gamma, \gamma \bmod \mathfrak{b}^{-1}} E(-\mu \gamma)+O\left(\frac{1}{(\log N)^{\sigma_{2 n / 2}}}\right) .
\end{aligned}
$$

By (4.4), (4.5), (4.8) and (4.9) we finally obtain

$$
\begin{aligned}
& R\left(\mu ; \lambda_{1}, \lambda_{2}, \ldots, \lambda_{s}\right) \\
& =\frac{1}{W^{s}} \Im_{G}(\mu) \Psi_{1}\left(\mu ; \lambda_{1}, \lambda_{2}, \ldots, \lambda_{s}\right) \prod_{k=1}^{s} N\left(\lambda_{k}\right)+O\left(\frac{N^{(s-1) n}}{(\log N)^{s+1}}\right) \\
& =\frac{\Psi_{1}\left(1 ; a_{1}, a_{2}, \ldots, a_{s}\right)}{W^{s}} \Im_{G}(\mu) \prod_{k=1}^{s} N\left(a_{k}\right) N(\mu)^{s-1}+O\left(\frac{N^{(s-1) n}}{(\log N)^{s+1}}\right) .
\end{aligned}
$$


This completes the proof of Theorem 2.

\section{REFERENCES}

[1] O. KöRNER, Erweiterter Goldbach-Vinogradovscher Satz in beliebigen algebraischen Zahlkörpern, Math. Ann., 143 (1961), 344-378.

[2] O. Körner, Zur additiven Primzahltheorie algebraischer Zahlkörper, Math. Ann., 144 (1961), 97-109.

[3] T. Mitsui, Generalized prime number theorem, Japan J. Math., 26 (1956), 1-42.

[4] T. MitsuI, On the Goldbach problem in an algebraic number field I, II, J. Math. Soc. Japan, 12 (1960), 209-324, 325-372.

[ 5 ] T. MitsuI, Analytic number theory, Iwanami, 1989 (in Japanese).

[6] H. Rademacher, Über eine Erweiterung des Goldachschen Problems, Math. Z., 25 (1926), $627-657$.

[7] H. Rademacher, Zur additiven Primzahltheorie algebraischer Zahlkörper III, Über die Darstellung totalpositiver Zahlen als Summen von totalpositiven Primzahlen in einem beliebigen Zahlkörper, Math. Z., 27 (1928), 321-426.

[ 8 ] C. L. SIEGEL, Generalization of Waring's problem to algebraic number fields, Amer. J. Math., 66 (1944), 122-136.

[9] C. L. SiEgel, Sums of $m$-th powers of algebraic integers, Ann. of Math., 46 (1945), 313-339.

[10] A. A. Sultanova, The Vinogradov-Goldbach theorem with restrictions on the terms, Izv. Akad. Nauk- UzSSR, no. 4 (1989), 37-39 (in Russian).

[11] R. C. Vaughan, The Hardy-Littlewood Method, Tracts in Math, 80, Cambridge Univ. Press, 1981.

[12] I. M. Vinogradov, Representation of an odd number as a sum of three primes, Dokl. Akad. Nauk SSSR, 15 (1937), 169-172.

[13] I. M. Vinogradov, Some theorems concerning the theory of primes, Mat. Sb., 2 (1937), 179195.

[14] Y. WANG, Diophantine Equations and Inequalities in Algebraic number Fields, Springer, 1991.

Department of Mathematics

FACUlty OF SCIENCE

ToKyo InStitute of TechNology

Oh-OKayama, Meguro-KU, ToKyo 152

JAPAN 